\title{
Application of Infrared Thermal Imaging in a Violinist with Temporomandibular Disorder
}

\author{
M. Clemente, MSc, D. Coimbra, PhD, A. Silva, MSc, J. Gabriel, PhD, C. Aguiar Branco, MD, \\ and J.C. Pinho, PhD
}

Temporomandibular disorders (TMD) consist of a group of pathologies that affect the masticatory muscles, temporomandibular joints (TMJ), and/or related structures. String instrumentalists, like many orchestra musicians, can spend hours with head postures that may influence the biomechanical behavior of the TMJ and the muscles of the craniocervicomandibular complex (CCMC). The adoption of abnormal postures acquired during performance by musicians can lead to muscular hyperactivity of the head and cervical muscles, with the possible appearance of TMD. Medical infrared thermography is a non-invasive procedure that can monitor the changes in the superficial tissue related to blood circulation and may serve as a complement to the clinical examination. The objective of this study was to use infrared thermography to evaluate, in one subject, the cutaneous thermal changes adjacent to the CCMC that occur before, during, and after playing a string instrument. Med Probl Perform Art 2015; 30(4):251-254.

$\mathrm{T}$ emporomandibular disorders (TMD) consist of a group of pathologies that affect the masticatory muscles, temporomandibular joints (TMJ), and/or related structures. The signs and symptoms referred by the patient are usually pain, joint sounds, muscle tenderness, joint tenderness, and limitation of jaw movement. Associated with these clinical situations, other symptoms can appear that affect the head and neck region, such as headache and earache. ${ }^{1,2}$

String instrumentalists, like many orchestra musicians, can spend many hours with head postures that may have an influence on the biomechanical behavior of the TMJ and the muscles of the craniocervicomandibular complex (CCMC)..$^{3,4}$ The adoption of abnormal postures acquired

M. Clemente, Dr. Branco, and Prof. Pinho are from the Faculty of Dentistry, University of Porto; Prof. Coimbra is from the School of Music and Performing Arts; and A. Silva and Prof. Gabriel are from Labiomep, INEGI, Faculty of Engineering, University of Porto, Porto, Portugal.

The authors declare no funding or conflicts of interest.

Address correspondence to: Prof. Joaquim Gabriel, Faculty of Engineering, University of Porto, Rua Dr. Roberto Frias, 4200-465 Porto, Portugal. jgabriel@fe.up.pt. during performance by musicians can lead to muscular hyperactivity of the head and cervical muscles. During playing of a string or a wind instrument, the musician's musculoskeletal system and head posture have a close relation with the orofacial anatomic zones. Some studies report the relation of head and cervical posture with the possible appearance of TMD. ${ }^{5}$ Given the duration and intensity of the daily practice of orchestra musicians, they may well develop a parafunctional activity, especially in the masticatory and postural muscles.

Medical infrared thermography can be used as an auxiliary tool in the field of sports medicine to investigate overuse and traumatic knee injuries, which can be used for preparticipation evaluation of athletes. ${ }^{6}$ Infrared thermography has the advantage of being a non-invasive procedure that can monitor the changes in the superficial tissue related to blood circulation. What is actually happening is a vasodilator thermoregulatory response caused by localized elevation of the body temperature and resultant heat loss through the skin. The assessment of surface temperature changes as a function of shoulder angle in relation to different work demands can be monitored by infrared imaging. ${ }^{\text {? }}$ The improvement of infrared thermographic devices has provided qualitative information on the temperature distribution of a chosen anatomic structure, like the TMJ. Studies have shown a low level of symmetry in TMD patients, when comparing the thermal patterns between the left and right TMJ. ${ }^{8}$ As the level of TMJ pain increases in the patients, the relative temperature (left vs right) of the TMJ also increases. ${ }^{8}$ Thermographic images with a difference of $0.36 \square \mathrm{C}$ or greater, left versus right side, have a positive correlation with a pathophysiological condition. ${ }^{9}$

Any coordinated neuromuscular task such as practicing up to $6 \mathrm{hrs} /$ day can eventually lead to the development of a variety of musculoskeletal disorders. This is a matter of occupational health that should be a major concern for clinicians including dentists, who frequently treat patients with TMD. At this time, to the best of our knowledge, very few studies have been done in performing artists applying infrared thermography as a diagnostic tool for musculoskeletal disorders affecting the CCMC. ${ }^{10,11}$

Aim: The objective of this study was to use infrared thermography to evaluate, in one subject, the cutaneous 
thermal changes adjacent to the CCMC that occur before, during, and after playing a string instrument.

\section{MATERIALS AND METHODS}

This study was approved by the Ethics Committee of the Dentistry University of Oporto, Portugal, and informed consent was obtained from the participant.

The musician is a 32-year-old female violinist who was referred to us due to her complaints regarding the CCMC. She plays in an orchestra, with rehearsals of $5 \mathrm{hrs} /$ day and 2 concerts per week. She has been playing her instrument for the past 25 yrs.

The musician was acclimated to the musical rehearsal room where the examination was done, with a resting period of 20 minutes before thermographic measurements were taken. Thermal images were obtained using the Flir ${ }^{\circledR}$ A325 thermographic camera. The thermographic patterns were analyzed with the ThermaCAM Researcher Professional software analysis system (ver. 2.10, Flir Systems AB, Sweden).

After thermal images were captured, a clinical assesment was conducted using a standardized clinical protocol that included an evaluation of the musician's history for any kind of discomfort or pain regarding the CCMC, palpation of the TMJs, auscultation of joint noises, palpation of the masticatory and postural musculature, and measurement of the mandibular range of motion.

The orofacial region of the violinist that was the main area of interest had left and right thermograms made in a rest position. In addition, dorsal and lateral right thermograms were taken during her musical performance. The thermal camera was mounted on a tripod and a digital camera was also present to monitor the repetitive movements of the musician while performing in order to understand the hyperactivity of specific muscles of the CCMC related to each particular instrumental technique (Fig. 1).

\section{RESULTS AND DISCUSSION}

Infrared thermography can be added to the existing types of imaging modalities for TMD, where the gold standard for studying TMD, especially in the cases of internal derangement, is magnetic resonance imaging (MRI), since it allows direct visualization of the articular disc. Nevertheless, some symptomatic TMJs show no sign of disc displacement on MRI examination. ${ }^{12}$ This can occur because referred pain from the masticatory muscles or postural muscles (like the upper trapezius) can induce pain that spreads to the head, causing symptoms perceived by TMD patients. In these cases, one can have myofascial pain, where most of the time the referred pain originates in some of the CCMC muscles. ${ }^{13}$ Therefore considering the functional aspects of the CCMC, diagnostic tools should not be limited to clinical and radiographic examinations.

Surface electromyography (sEMG) can be considered as a safe and effective method of monitoring muscles in

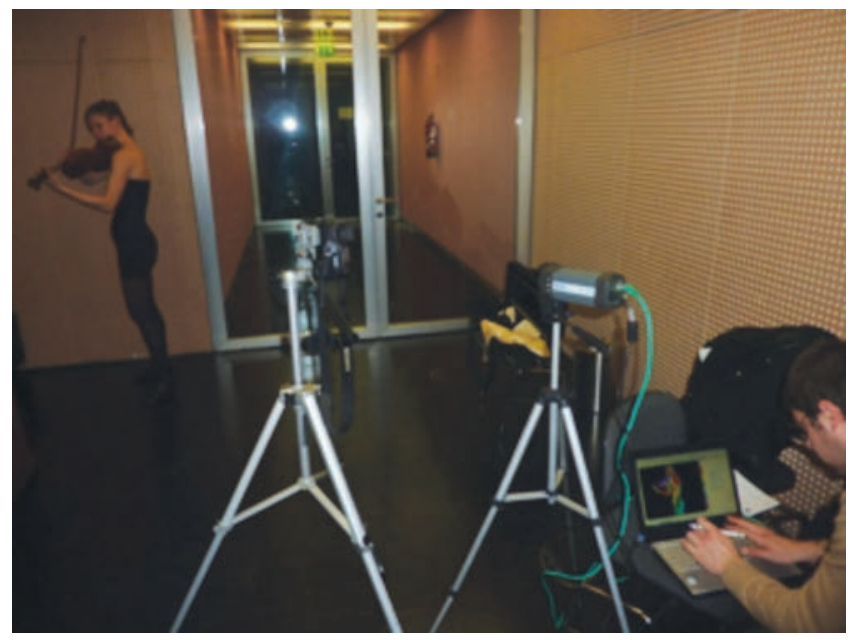

FIGURE 1. Violinist during warm-up in the rehearsal room where the infrared thermography examination occurred.

healthy and dysfunctional individuals. ${ }^{14}$ In the dental field, research with sEMG has been applied since late $1940 \mathrm{~s},{ }^{15}$ where its purpose was to create a quantitative measurement of the amount of dysfunction present in the masticatory system when compared with healthy or asymptomatic function. Nevertheless there are factors that can cause variation in these measurements, such as technical and biological factors, especially recording technique and muscle variables, among others. ${ }^{16}$ As a result, sEMG analysis of TMD patients has some limitations, although it is still important as an a complementary research tool.

In this study, we have demonstrated that infrared thermography can be a complement to a clinical examination of the CCMC, in order to fully assess the cutaneous layers adjacent to the masticatory and postural muscles. Parafunctional muscular activities that are part of the multifactorial etiology of TMD can be monitored by thermographic cameras during the performance of various tasks, in this particular case during musical performance. With the thermographic pattern, dorsal view, obtained with this violinist, it was possible to observe the temperatures corresponding to the trapezius zone during her musical activity (Fig. 2). The thermograms helped to explain what is happening in her body, which has two distinct movements of the upper extremities. Her left arm is isometrically contracted in order to hold the violin between her shoulder, chest, and mandible, while the right arm is exerting an isotonic contraction with the violin bow, playing the musical notes. Eventually, biomechanical loading of certain muscular groups of the CCMC can lead to overload and/or fatigue of the muscles involved and structures of the orofacial region.

With infrared thermography, it was possible to observe specific areas of interest in the CCMC, such as the sternocleidomastoid, which has high temperature values when compared with other sites (Fig. 3). String instrumentalists such as violin or viola players often have neck tenderness, discomfort in the jaw, or pain in the TMJ. These symp- 


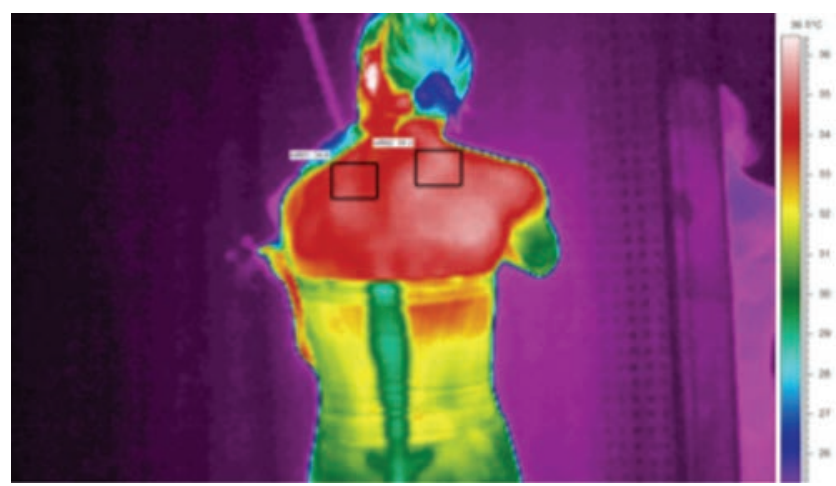

FIGURE 2. Initial dorsal thermal image of the violinist, showing similar temperature values comparing the left and right side trapezius, $34.6^{\circ} \mathrm{C}$ and $34.8^{\circ} \mathrm{C}$ respectively. 。

toms are frequently associated with parafunctional activities that occur while playing the violin, such as clenching of the teeth, with consequent changes in the temperature of the masseter muscle area.

This violinist's clinical findings correlated with the lateral thermograms of the face, where the presence of muscular hyperactivity of the elevator muscles caused higher temperatures in the area of the masseter muscle. The major and only complaint of this violinist player was soreness on the left side of her face involving the masseter region. Her clinical examination revealed tenderness in the left masseter muscle. The thermograms allowed us to visualize an asymmetric pattern of this anatomic region involving the masticatory muscles. The masseter muscle serves primarily to elevate the mandible and causes a slight protrusion, which is in accordance to the role and action of the mandible to stabilize the violin during the violinist's performance. Usually in this situation, the violinist's mandible deviates laterally to the left side and anteriorly. The complexity of this action implies that clenching of the teeth is common and is associated with muscular hyperactivity mainly of the masseter muscles. This isometric con-

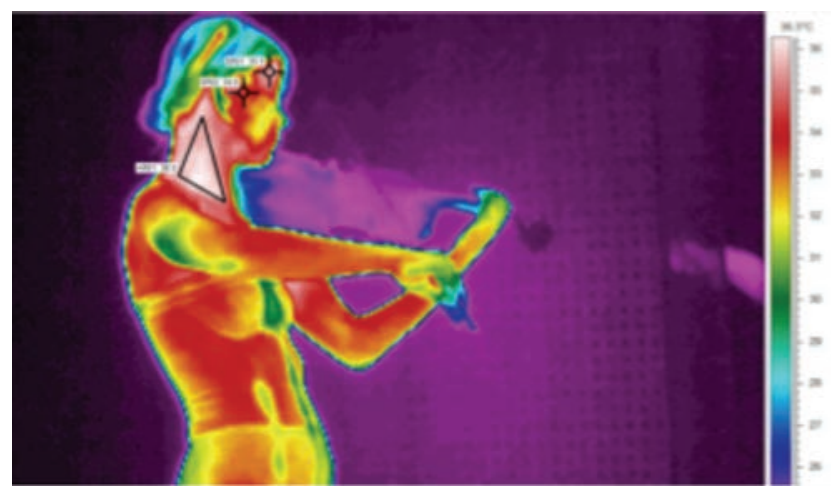

FIGURE 3. Thermal image of identified zones such as the sternocleidomastoid, TMJ, and the anterior temporalis muscle.

traction induced higher temperatures on the left masseter muscle of the instrumentalist, with a temperature difference of $0.9^{\circ} \mathrm{C}$ (Fig. 4).

These thermal images can serve as a complementary examination to the clinical evaluation of the musician. Nevertheless, it is of vital importance to mention that thermograms will not give clinicians a diagnosis. The most important procedure when observing a musician who is complaining of pain is the clinical examination and a complete history. The thermographic examination can be a very important tool for visualization of the musculoskeletal system of the musician, in order to understand if there are specific muscular groups that are being used more than others. It is known that thermography is able to detect the surface temperature of a human body and that this temperature corresponds to physiological activities that are happening underneath the skin, mainly in the muscles. So when an inflammatory process involves an articular or muscular zone due to the occurrence of repetitive movements, we can expect to observe thermograms with higher temperatures involving these regions. Using thermal imaging as a complement to the clinical examination, it may be
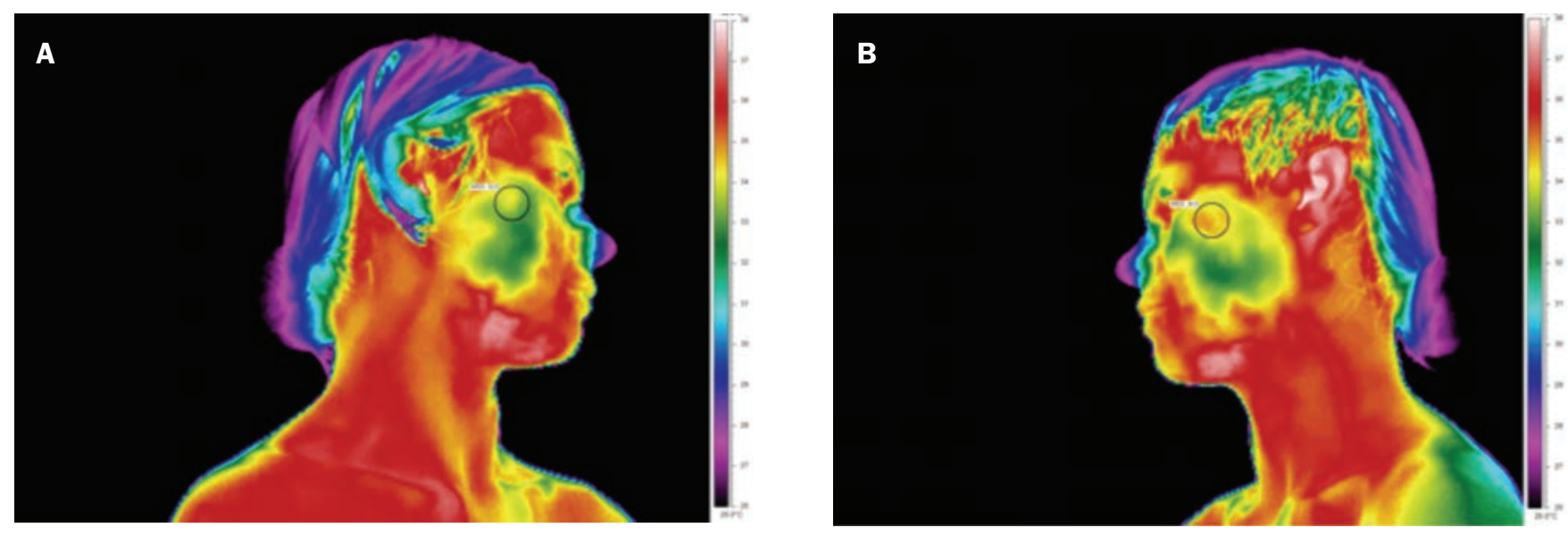

FIGURE 4. Initial orofacial thermal images: $\mathrm{A}$, On the right side, the masseter region temperature is $33.6^{\circ} \mathrm{C}$. $\mathrm{B}$, On the left side, the masseter region temperature is $34.5^{\circ} \mathrm{C}$, making it possible to identify an asymmetric pattern compared to the right side. 
possible to detect signs before they become complaints. This actually allows the clinician to observe thermograms of areas where pain can be present or will be present in the near future due to overuse of certain muscles.

The usefulness of thermography in performing arts medicine, in this particular case for the evaluation of TMD, is that this technique allows us to evaluate an orchestra musician "from invisible to visible," which means that on the musician's cutaneous surface, one can actually visualize what is happening inside the body during performance.

\section{REFERENCES}

1. Okeson Jeffrey P. Management of Temporomandibular Disorders and Occlusion, 6th ed. Mosby Elsevier, 2008.

2. Dawson Peter E. Functional Occlusion: from TMJ to Smile Design. Mosby Elsevier, 2007.

3. Clemente M, Vasconcelos M, Pinho JC, et al. Dental considerations and electromyographic study of orofacial muscle activity in musicians playing wind and string instruments. In Proceedings of Music, Health and Happiness Conference, Royal Northern College of Music, Manchester, 6-8 Nov 2008.

4. Clemente M, Coimbra D, Silva A, et al. As diferentes embocaduras dos instrumentistas de sopro e as suas implicações a nível do complexo crânio-cérvicomandibular. Livro de actas de conferências do $4^{\circ}$ Congresso Nacional de Biomecânica, Sociedade Portuguesa de Biomecânica, Coimbra, 2010.

5. Armijo-Olivo S, Rappoport K, Fuentes J, et al. Head and cervical posture in patients with temporomandibular disorders. J Orofacial Pain 2011;25:199-209.

6. Hildebrandt C, Raschner C, Ammer K. An overview of recent application of medical infrared thermography in sports medicine in Austria. Sensors 2010;10:4700-4715.

7. Bertmaring I, Babski-Reeves K, Nussbaum MA. Infrared imaging of the anterior deltoid during overhead static exertions. Ergonomics 2008;51(10):1606-1619.

8. Canavan D, Gratt BM. Electronic thermography for assessment of mild and moderate temporomandibular joint dysfunction. Oral Surg Oral Med Oral Pathol 1995;79:778-786.

9. Gratt BM, Graff-Radford SB, Shetty V, et al. A 6-year clinical assessment of electronic facial thermography. Dentomaxillofac Radiol 1996;9:255-265.

10. Clemente $\mathrm{M}$, Coimbra D, Silva A, et al. Can infrared thermography be a diagnostic tool for myofascial pain in wind and string instrument players? In Proceedings of the International Symposium of Performance Science 2011, Toronto, Canada.

11. Lourenço S, Clemente M, Coimbra D, et al. The assessment of trapezius muscle symptoms of piano players by the use of infrared thermography. In Proceedings of the International Symposium of Performance Science 2011, Toronto, Canada.

12. Emshoff R, Brandlmaier I, Gerhard S, et al. Magnetic resonance imaging predictors of temporomandibular joint pain. J Am Dent Assoc 2003;134(6):705-714.

13. Svensson P, Graven-Nielsen T: Craniofacial muscle pain: review of mechanisms and clinical manifestations. J Orofacial Pain 2001;15:117-145.

14. Svensson P, Wang K, Sessle JB, Arendt-Nielsen L. Associations between pain and neuromuscular activity in the human jaw and neck muscles. Pain 2014;109:225-232.

15. Moyers RE. Temporomandibular muscle contraction patterns in angle class II division I malocclusions: an electromyographic analysis. Am J Orthod 1949;35:837-857.

16. Klasser GD, Okeson JP. The clinical usefulness of surface electromyography in the diagnosis and treatment of temporomandibular disorders. J Am Dent Assoc 2006; 137:763-771. 